\title{
EL USO ADECUADO DE LA GESTIÓN DEL TALENTO
}

\section{THE PROPER USE OF TALENT MANAGEMENT}

\author{
Catalina Abarca' Efrain Flores ${ }^{2}$
}

\begin{abstract}
Resumen
Para cubrir adecuadamente el objetivo del artículo, es necesario convenir en que el término talento humano es inapropiado, considerando que solo los humanos tienen talento. El planteamiento puntualiza, que las organizaciones que aspiren desarrollar efectivamente sus talentos, deberán sustentar sus esfuerzos en acciones como: contar con candidatos calificados, implementar un buen clima laboral, comunicación adecuada, orgullo de pertenencia, proveer a los colaboradores de autonomía para gestionar, plantear nuevos retos, subsistema objetivo de evaluación del desempeño y, por último, gratificaciones que retengan los mejores talentos. Para ello se realizaron búsquedas bibliográficas relacionadas al tema, a efectos de contrastar la aplicación empírica de esta gestión, con la aplicación de las acciones propuestas requeridas, tanto a corto como a largo plazo. Los resultados apoyan la hipótesis planteada, por cuanto es facultad de la administración de las organizaciones, a la hora de tomar decisiones, el garantizar la aplicación de un proceso metodológico, tomando como fuente de información bibliográfica otros artículos inherentes al tema, combinados con investigación de campo a través de consulta a expertos, y planeado para llevar a cabo con éxito una estrategia de gestión en pro del desarrollo organizacional. Este aporte se sustenta en una investigación que recopila y organiza datos bibliográficos. Se debe concluir que el elemento más importante de la organización es un talento empoderado, que sienta que su aporte es su proyecto de vida. El éxito o fracaso de las organizaciones depende del capital humano con que cuente.
\end{abstract}

\section{Palabras clave}

Talento, gestión, desarrollo organizacional, motivación, estrategias.

\begin{abstract}
In order to properly cover the article's objective, it is necessary to agree that the term human talent is inappropriate, considering that only humans have talent. The approach points out that the organizations who aspire to effectively develop their talents, will have to sustain their efforts in actions such as; counting with qualified candidates, implementing a good work environment, adequate communication, pride of belonging, to provide their collaborators with autonomy to manage, stablishing new challenges, having an objective subsystem of performance evaluation and, lastly, rewards which retain the best talents. For this, bibliographic researches inherent to the topic were made, with the purpose to contrast the empirical application of this work, with the application of the required proposed actions, both in short, and in long time. The results support the posed hypothesis, for what is faculty of the organizations' administration at the time of making decisions, to guarantee the application of a planned methodological process taking as source of bibliographic information other articles inherent to the topic combined with field research through consultations with experts planned to successfully implement a management strategy in pro of the organizational development. This input is sustained on an investigation that collects and organizes bibliographic data. It should be concluded that the most important element in the organization is an empowered talent who feels that their help is their life project.
\end{abstract}

\section{Keywords}

Talent, management, organizational development, motivation, strategies. 


\section{Introducción}

Para (Freedman,2004) el papel de las personas en la sociedad del conocimiento, es fundamental pues está inmerso, se transmite, se crea y son valorados por sus conocimientos, sus aportes intelectuales y su talento en la consecución de las metas de las organizaciones. Para implicar a las personas en la organización, no basta con movilizar sus recursos intelectuales, físicos o de interrelación, esperando incrementos de productividad, lo que se requiere es comprometer a las personas como sujetos de su personalidad en relación con el trabajo y la organización. (Ferrer, A. 2008)

Como punto de partida, es necesario aclarar que este aporte no se centra en la gestión del talento humano, sino más bien se refiere específicamente a la gestión del talento, este último definido como "aptitud, capacidad para desempeñar o ejercer una actividad, vinculado a la aptitud o la inteligencia". Se trata de la capacidad para ejercer una cierta ocupación o para desempeñar una actividad. El talento suele estar asociado a la habilidad innata y a la creación. La especial capacidad intelectual o aptitud que una persona tiene para aprender las cosas con facilidad o para desarrollar con mucha habilidad una actividad, es potestad de los humanos; bajo esa concepción, decir talento humano sería redundante.

El talento es un término utilizado para referirse a esa cualidad particular que puede poseer una persona en un campo específico, debido a que se desenvuelve con gran facilidad en ello. De alguna manera una persona talentosa es capaz de explotar su máximo potencial en la realización de la labor que mejor desempeña. En la mayoría de los casos estas aptitudes se manejan en las áreas artísticas e intelectuales, en las que se desenvuelve una persona y que, gracias a sus posibilidades, puede destacarse en aquello que domina.
La gestión de talento demanda centrarse en el valor de las personas, lo cual implica aplicar una relación estratégica de mayor acercamiento, para lograr conocerlas mejor y sacarles mayor partido a sus fortalezas, así como ayudarles a reforzar en la superación de sus debilidades. El objetivo de este aporte es conocer de primera mano el uso adecuado del talento.

Según expertos (Universidad de Sevilla, Profesionales 2020), el talento es descrito como la característica de aquellas personas cuyas capacidades se encuentran comprometidas a hacer las cosas que mejoren los resultados de una organización.

(Vivas, E 2010). El entorno actual se caracteriza por un mercado con alta competitividad, globalizado e incierto, que demanda calidad, innovación y externa atención al cliente. Las organizaciones se han visto afectadas por este constante cambio y con ello el recurso humano de las mismas.

Si se analizara desde el punto de vista empresarial, diríamos que se debe tener en cuenta diversos factores, que influyen en el grado de satisfacción del empleado y están relacionados con su puesto de trabajo. Esto abarca desde gustos e intereses, hasta determinar las necesidades de esparcimiento, descanso, reconocimiento, autorrealización, socialización y remuneración.

La experiencia laboral describe el talento como característica de personas cuyas capacidades se encuentran comprometidas a mejorar los resultados de la organización. Por tanto, estamos ante individuos que ponen en ejecución sus capacidades para obtener resultados superiores. No obstante, se puede ser inteligente y apto sin ser talentoso. Es necesario advertir que la definición de talento está estrechamente asociada a la generación de valor.

Por su parte Goleman, D.(2010) afirma que las reglas de trabajo están cambiando, ahora se juzga según normas nuevas: que ya 
no importa solo la sagacidad, la preparación y la experiencia sino como nos manejamos con nosotros mismos y con los demás. Es por eso que es importante tomar en cuenta algunas consideraciones sobre la gestión del talento humano.

Para el desarrollo del artículo se ha tomado, como fuente de información bibliográfica, otros artículos relacionados al tema, que van reforzados con investigación de campo, a través de consulta a expertos, entre los cuales mencionan: "Las personas actúan en la organización en función de sus expectativas y la fuerza de convicción en la consecución de las metas que poseen y desean alcanzar" (Gillen \& Guil, 2007)

Con estas aclaraciones y precisiones en el desarrollo de este trabajo, surge la necesidad de introducir al lector en el manejo adecuado del talento organizacional.

\section{Fundamentación teórica}

Bajo las consideraciones expuestas, sería importante considerar otros componentes claves, que dan valor agregado a la propuesta.

- Capacidad. Considerada como un conjunto de conocimientos, actitudes y competencias que se deben desarrollar dentro de la organización.

- Compromiso. Definido como el grado de empoderamiento hacia la organización, a través del esfuerzo invertido en el trabajo.

- Acciones. Actuaciones y conductas llevadas a cabo dentro de una organización, intermediadas por el compromiso interno con la misma y las capacidades del propio individuo.

- Empatía. Habilidad para atender las necesidades, los sentimientos y problemas de los demás, poniéndose en su lugar y respondiendo correctamente a sus reacciones emocionales. Madrigal, B.(2009).
- Motivación. Ligada a la idea de que las personas con talento acaban realizando lo que hacen y que disfrutan de ello, condición enlazada con la pasión.

- Herramientas de conocimientos. Adquisición y renovación de los saberes que se encuentran anclados con el desempeño de aquellas tareas que nos gustan.

- Circunstancias. Condiciones externas determinadas e idóneas que promueven el desarrollo. Un claro ejemplo serían aquellas organizaciones no basadas en la filosofía del control. (Sukier, H., Molina R., Parra M, Cueto, K.2020).

Para reforzar lo anterior y justificar el planteamiento, se requiere potenciar los talentos, a través de las siguientes estrategias:

- Evalúa en lo que eres bueno y conoces a profundidad: Analiza tus puntos fuertes y aquellas competencias que manejas de manera positiva, y piensa cómo podrías extrapolarlas al entorno organizativo.

- Piensa en aquellos momentos en que hayas tenido éxito: Haz uso de la memoria sobre tu propia experiencia, para tener en claro cuáles son las capacidades que han configurado tus logros.

- Desarrolla las habilidades que ya tengas: Haz uso de tus mejores destrezas y transfiérelas al campo profesional.

- Actualízate en los temas que te interesen: Si aún piensas que necesitas mejorar, haz uso de la formación teórica y práctica. El talento también necesita actualizarse.

- Hazte cargo de los desafíos: Busca oportunidades para aplicar tu talento y ponte a prueba en aquellas situaciones que impliquen iniciativa y proactividad.

- Apuéstale a la innovación: Debes diferenciarte de tus competidores 
dando de ti todo lo mejor. Sé muy dinámico y sobre todo siempre enfócate en un proceso constante de actualización de conocimientos.

\section{- Fomenta tu motivación personal:} No solo esperes que el proceso de motivación venga desde tu empleador a través de un adecuado ambiente de trabajo, una remuneración justa o simplemente los beneficios sociales que no deben faltar; es hora de mirar más allá. El sentirte automotivado todos los días, el tener gusto por lo que haces, te va a facilitar mucho los procesos y vas a llegar a la meta que te propones.

- Valora tus logros obtenidos: En muchas empresas se olvidan de reconocer el buen trabajo que hayas aportado, sin embargo, continúa esforzándote más, para que demuestres en tu equipo de trabajo la calidad profesional que te caracteriza; aplica todos los valores inculcados por herencia en tu hogar.

- Mide a diario tu rendimiento: Siéntate tranquilamente y analiza después de tu larga jornada cuáles fueron tus logros $y$, si no terminaste algún proceso, no te desesperes, mantén la calma; piensa detenidamente en el aporte que has dado en ese día y utiliza estrategias para el logro de tus objetivos.

- Globalízate: Hoy en día la creciente complejidad de la tecnología de la información, que va de la mano con la inestabilidad del mercado laboral, te alerta a generar y desarrollar nuevas competencias que aporten valor a tus conocimientos.

- Lealtad: La experiencia y los años son un factor de gran ventaja competitiva, que te hace madurar en todo sentido.

- Dale importancia a la perseverancia: Sé constante y lucha por tus ideales: todo esfuerzo requiere de mucho sacrificio.
Según (González, T.; Martínez, C.; Pardo Del Val, M. 2009) la gestión del talento es considerada por algunos como un compendio de mejores prácticas en la gestión de recursos humanos, o de una apuesta por desarrollar al máximo la búsqueda de los mejores empleados y su dirección, la gestión del talento va más allá, a partir de la relación entre la dirección de recursos humanos y la estrategia empresarial.

Hoy en día la gestión del talento es un reto para todas las organizaciones, pues el aporte de valor se produce cada vez más desde el área del capital intangible con el cual cuentan todas las organizaciones. Es muy claro que la gestión del capital humano es una palanca de alto rendimiento, en donde todos ganan, tanto empleadores como trabajadores. Por otro lado, se puede identificar una serie de procesos esenciales que debe incorporarse en todo plan de talento: atracción, selección, identificación, desarrollo y retención. Por esto, la gestión del talento ha experimentado cambios e innovaciones en los últimos años, como producto de una evidente necesidad de establecer un sistema de gestión del talento, como medio para optimizar el rendimiento de cada colaborador y de la organización. Sin embargo, en muchas empresas el concepto de la gestión del capital humano apenas empieza a desarrollarse.

A partir del siglo XX y más aún en el siglo XXI, la mayoría de las empresas han tenido que diseñar e innovar su gestión, y aceptar que las personas juegan un papel muy importante en las organizaciones, pues es en el interior de estas que se dinamizan los procesos de aprendizaje, se generan conocimientos y adaptaciones rápidas a cambios organizacionales, necesarios para lograr mayor competitividad y sostenibilidad.

El desarrollo organizacional, por ejemplo, ha sido un esfuerzo de largo plazo, apoyado por la alta dirección, que tiene por objeto mejorar los procesos para la solución de 
problemas y la renovación organizacional. Esto, a través de un diagnóstico eficaz, en colaboración con la administración de la cultura organizacional, con especial importancia en los equipos formales de trabajo, los equipos temporales y la cultura intergrupal, que cuentan con la ayuda de un consultorfacilitador y la aplicación de la teoría y la tecnología de las ciencias de la conducta, inclusive la acción y la investigación (Chiaveanato, 2013). Se puede colegir que este punto de vista se sustenta en el talento (personas), que conforma la organización.

Existe una serie de elementos acerca de la categoría de talento que no se deben ignorar: ¿Qué es el talento y cuál es su relación con categorías como inteligencia, creatividad, conocimiento, competencias? ¿Cuáles son las condiciones sociales del talento? ¿Cuál es la relación entre el talento y la edad? ¿Qué implicaciones tiene para el talento el área del conocimiento a la que se dedica el sujeto? ¿Cómo las personas pueden autodesarrollar su talento? (Chiaveanato, 2013).

Las organizaciones cada vez toman nuevas formas y siempre están adaptándose a los escenarios cambiantes que viven en el día a día, y que les obliga a una continua transformación en los procesos de la gestión del talento. En la actualidad toda organización busca gente profesional con una gran ductilidad, que sea capaz de integrarse en equipos autónomos y proponga metodologías ágiles para el desarrollo de sus procesos.

La consideración de estas cuestiones y cómo ha sido estudiada esta categoría, desde la óptica de diferentes ciencias, es importante porque no se puede gestionar un fenómeno que no se domina, que no se sabe lo que es. La advertencia es importante porque, en ocasiones, se confunde la gestión del talento con la gestión del conocimiento, con la gestión de competencias y con la gestión de los recursos humanos o del capital humano en general; es más, la gestión del talento también es conocida como gestión del capital humano, sistema de información del recurso humano o módulos de recursos humanos.

Por lo tanto, es imprescindible insistir sobre el tema, ya que al referirse al manejo pragmático y, más que nada, consciente de las personas que forman parte de un escenario tan complejo como la empresa, debe existir el pleno convencimiento de lo que estamos gestionando.

La revista Gestión del capital Humano y del talento humano (www.gestiopolis. com/gestion-del-capital-humano-y-del-talento-humano) menciona que la gestión del talento se refiere "al proceso que desarrolla e incorpora nuevos integrantes a la fuerza laboral, y que además desarrolla y retiene a un recurso humano existente" (Rodríguez Cruz, 2021).La gestión del talento busca básicamente ubicar a aquellas personas con un alto potencial, para la función que les ha sido encomendada; además, intenta retener 0 atraer a aquellas personas con talento. Este proceso se ha tornado cada vez más competitivo entre las empresas y tiene prioridad estratégica en la búsqueda permanente del cumplimiento efectivo de los objetivos organizacionales. La gestión del talento, como proceso, surgió recién en los años noventa, cuando las empresas hicieron conciencia de que, lo que impulsa el éxito de su negocio, son el talento y las habilidades y destrezas del cliente interno. Como dato referencial, en el Ecuador, ventajosamente, a partir de los años referidos se da inicio a una verdadera revolución para revertir aquello de que la gestión del talento debía ser cosa de cualquier mortal dispuesto a manejar subprocesos empíricos tales como: el control de asistencia, vacaciones, permisos, licencias, disciplina, pagos a través de lo que se llamaba el "parte de novedades", cursos, talleres y seminarios sin planificación previa, entre otros. Era impresionante ver cómo se ponían 
al frente de esta gestión, exmilitares, economistas, sociólogos, psicólogos, abogados $y$, aunque parezca mentira, individuos sin la formación mínima necesaria para afrontar el manejo de talentos y peor aún resolver sus problemas, o por lo menos disminuirlos.

El tema es que muchas organizaciones hoy en día realizan un enorme esfuerzo por atraer colaboradores a su empresa, pero descuidan su retención y desarrollo, ya que se deja esta labor en manos únicamente del departamento de recursos humanos, cuando esta es una práctica que debería ser aplicada en todos los niveles de la organización. Las unidades, dentro de la compañía, deben compartir abiertamente la información con otras dependencias, para que los colaboradores logren el conocimiento pleno de los objetivos organizacionales.

\section{La gestión del talento}

El psicólogo alemán Albert Mehrabian (2018) concluyó en los años ochenta que, cuando comunicamos emociones y sentimientos, más del 90 \% del mensaje recae sobre la comunicación no verbal. La conocida regla de Mehrabian dice que en una conversación personal o íntima, solo un $7 \%$ de lo que recibe nuestro interlocutor proviene de lo que hemos dicho con la palabra. El 93 \% restante, proviene de lo que "no se ha dicho", de la comunicación no verbal. La regla 7-38-55 rige e indica, fundamentalmente, que en las conversaciones en donde entran en juego emociones y sentimientos, el mensaje que vamos a comunicar a nuestro público proviene, en un $55 \%$, de lengua corporal, a través de gestos y posturas; en $38 \%$ del lenguaje paraverbal, relacionado con la voz, el tono y la entonación; y, únicamente en $7 \%$ del lenguaje verbal, es decir las palabras que la persona expresa (Ávila, s.f.).

Las organizaciones que aspiren a gestionar y desarrollar efectivamente sus talentos, deberán sustentar sus esfuerzos en acciones tales como: atraer y reclutar candidatos calificados con formación competitiva, como potenciales colaboradores, para lo cual será necesario mantener salarios justos. A veces las remuneraciones no concuerdan con las horas laborales o con la cantidad o calidad de trabajo que realizan. Muchas veces no es incentivo aumentarlo para que el colaborador se mantenga en el puesto, lo importante es que la empresa pague salarios justos y competitivos; es decir, que estén conforme a su desempeño y a lo que ofrece el mercado laboral. En la actualidad ha cobrado auge el salario emocional ya que incide en la gestión estratégica del clima y fidelidad laboral. Estas deben ser algunas de las estrategias a considerar (Llácer, P. 27 de febrero de 2019):

- Vinculación. Es importante una adaptación favorable del empleado a la organización, puesto que debe familiarizarse con la cultura y las responsabilidades de su puesto de trabajo.

- Un buen clima laboral. Esto garantiza el respeto por los colaboradores y va de la mano con la lealtad para la empresa, lo cual quiere decir que mientras los empleadores entreguen una estación de trabajo personalizado, posibilidades ciertas de formación - desarrollo y un ambiente cálido-, las personas de hecho, serán más fieles con la empresa. Al mismo tiempo, este valor de respetar al otro, considera las relaciones interpersonales entre empleador y trabajador, pero también entre compañeros.

- Canales de comunicación adecuados y beneficiosos para las partes. Es preciso que la empresa informe periódicamente sobre las ventajas del empleo que mantiene. Esto puede ser transmitido por medio de grupos focales, siendo también útiles las encuestas de conformidad con los cargos dis- 
ponibles. De esta manera, la empresa puede centrar sus esfuerzos en remediar sus debilidades con los colaboradores y hacer que estas debilidades se conviertan en potenciales fortalezas. Paralelamente, es primordial contar con una estrategia de comunicación organizacional, donde constantemente se recuerde a los empleados los valores, misión, visión y objetivos de la empresa, así como los logros obtenidos por esta. Crear una identidad corporativa compartida es la mejor técnica para tener un personal contento con su labor; deben saber para qué y para quién trabajan.

- Escucha activa. En una conversación siempre mantener el contacto visual con la otra parte. Es necesario tener una buena actitud, mantener una postura corporal asertiva, sentirse cómodo con lo que se habla, prestar atención a lo que se dice.

- Orgullo de pertenencia. Los colaboradores desean trabajar en organizaciones bien gestionadas, con una imagen positiva de sus líderes como profesionales competentes. Eso implica tener una visión clara del futuro de la organización y de cuáles son las claves para lograr el éxito. Es importante, por tanto, que sepan transmitir la energía que se requiere para motivar a los equipos en la consecución de dicha visión.

- Proveer a su gente tanta autonomía como pueda gestionar. A muchos les gusta trabajar bajo poca supervisión. Si se les permite, son más felices y harán la vida de sus supervisores más fácil. Es conveniente también permitir, hasta donde sea posible, que tomen decisiones, sin olvidar que la esencia de una buena dirección es justamente la toma de decisiones en conjunción con habilidades conceptuales, técni- co-profesionales, interpersonales, intrapersonales, grupales, organizacionales y, desde luego, sociales.

- Plantear nuevos retos. A la mayoría de los colaboradores, a quienes se está tratando de retener, les encanta asumir nuevos retos y tener la sensación de que la organización confía en ellos para tomar responsabilidades de mayor nivel de las que podrían esperar. Es importante poner a la gente que más se desea retener, en posiciones que les supongan nuevos retos y darles el apoyo que necesiten para desempeñarse con éxito.

- Definir un proceso de evaluación del desempeño, que permita evaluar las actividades del trabajador, entendiéndose como éste cumple a satisfacción con las funciones de su trabajo para ayudar a elevar la productividad en las organizaciones. Este subproceso deberá servir de base para la administración de ascensos y traslados, considerando sobre todo las necesidades de la organización y de los colaboradores.

- Gratificaciones. Es primordial mantener a los empleados clave que pueden ayudar con buenos resultados a mantener el funcionamiento de la empresa. Se debe ofrecer a los colaboradores gratificaciones que los retengan, por ejemplo, en momentos de transición o de expansiones. Esas gratificaciones deben ser acordadas con el empleador como beneficios propios de un esfuerzo superior, no como un derecho sindical, ya que se distorsiona el ánimo de la gratificación cuando los sindicalistas consideran a esta como derechos adquiridos de por vida, haya o no un rendimiento superior a la media. Estos beneficios se refieren a transporte, educación, seguros inclusive para sus familias, mejoras en la 
jubilación a lo largo del tiempo de servicio, así como bonos extra.

- Desarrollo profesional. Se entiende como la capacidad de adquirir mayores niveles de competencias y de asumir mayores grados de responsabilidad y autonomía para todo progreso del ser humano.

Figura 1. Cultura corporativa

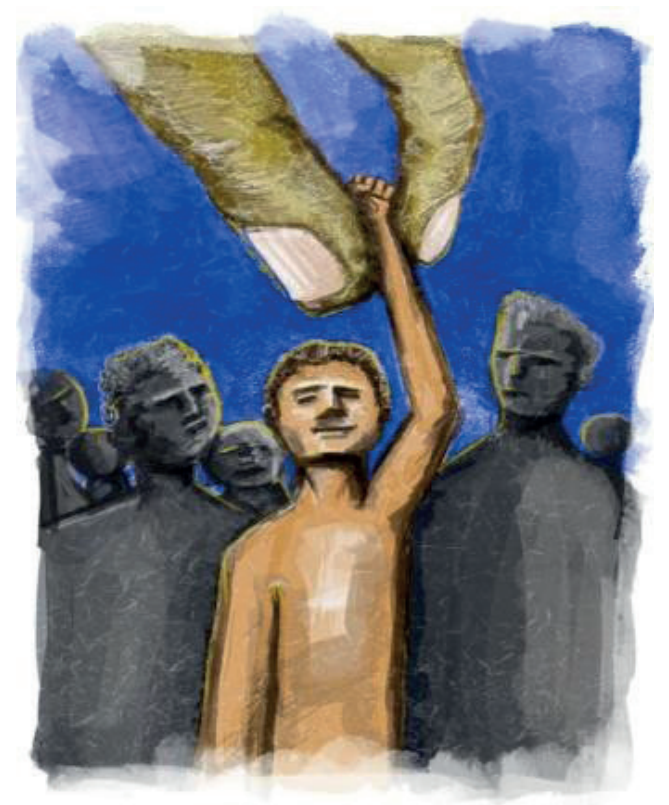

Cortesía: Flores García David Efrén.

Las investigaciones realizadas para medir el valor de estas acciones descubren beneficios en áreas económicas críticas: utilidades, satisfacción del cliente, calidad, productividad, costos, duración del ciclo de los procesos y capitalización de mercado. Este abordaje del manejo del talento busca no solo emplear al personal más calificado y valioso, sino también enfatizar en su retención.

De hecho, la gestión del talento desempeña un papel muy importante en la estrategia comercial, ya que gestiona el activo más importante que toda organización tiene. Hoy en día la gestión del talento abarca todas las áreas de la organización, sean estas administrativa u operativa, y siempre se está innovando con mejoras continuas, tomando en consideración las necesidades de un mercado competitivo.

Si hablamos de un mercado competitivo, haremos referencia a la movilidad de ese talento, como un proceso integral, en la gestión de habilidades que sustentan el movimiento de las personas que giran en torno a la capacidad de una organización, para entender, desarrollar y desplegar eficazmente el talento en respuesta a las necesidades de cada organización.

La gestión del talento siempre ofrecerá resultados que se verán registrados en los indicadores económicos clave, para no solo obtener ganancias esperadas en un periodo determinado, sino más bien, tener un buen grupo de talentos, cuyas habilidades necesarias, como el conocimiento, rasgos de comportamiento y sobre todo el potencial para hacer bien las cosas, ayuden a elevar la productividad en las organizaciones; esto a su vez les convertirá en sostenibles en el mercado meta.

Edward Deming establece un referente de la calidad y dice que, al mejorar la calidad de un producto, se genera mayor productividad, menores costos, menores precios; se crean nuevos mercados, existen mayores ganancias. Esto se traduce en un desarrollo integral, mejores oportunidades y, como resultado tangible, la elevación del nivel de vida. Pero lo interesante de esta propuesta (ciclo de la calidad), es que en todos sus componentes se encuentra inmerso el talento, creando calidad y en torno al cual gira el ciclo.

El objetivo fundamental de este artículo es la introducción del lector al campo de una administración moderna, en cuanto al uso adecuado de la gestión del talento en las organizaciones. En tal contexto es casi imposible separar cada comportamiento, aunque hasta hace poco la relación entre estas se consideraba antagónica y conflictiva, al creer 
que sus objetivos eran incompatibles. El escenario en que se sitúa la gestión del talento muestra que las organizaciones están conformadas por personas y que dependen de ellas el alcanzar objetivos y cumplir las misiones.

Figura 2. Espíritu de equipo

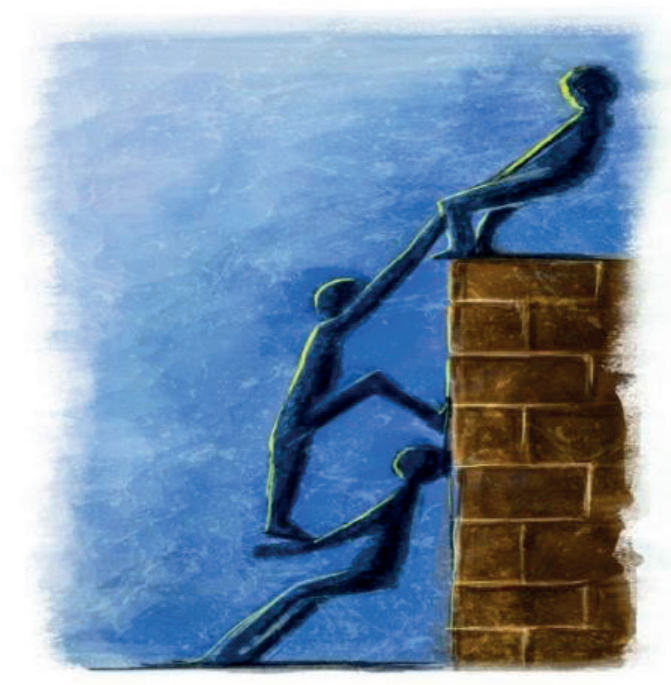

Cortesía: Flores García David Efrén.

En este sentido para (Bell, R., Espín, M., Espín,E 2015) es importante el talento humano, pues se considera el recurso más importante para el funcionamiento de cualquier organización. Si el elemento humano está dispuesto a proporcionar su esfuerzo, la organización marchará de manera eficiente, de lo contrario se detendrá, por otra parte la gestión del talento es el andamiaje administrativo en la toma de decisiones.

(Benítez, K. 2005) Ramón Piñango, en el editorial que escribiera para la Revista Debates IESA de Octubre-Diciembre 2004, apuntó: La realidad humana de las organizaciones ha conducido a unos cuantos especialistas a buscar formas de lidiar con la gente y convertirla en una fuerza a favor de la organización o, a menos, impedir que cause perturbaciones mayores. Cada organización es distinta, como somos distintos los seres humanos, puesto que, aunque se dediquen a una misma actividad, sus fundamentos y actitudes son particulares.

En la era actual del conocimiento y la innovación las organizaciones cumplen un papel fundamental, pero más aún las personas que las integran, pues son éstas quiénes al interior de las empresas dinamizan los procesos de aprendizaje, la generación de conocimiento y los cambios necesarios para lograr mayor competitividad y sostenibilidad organizacional. (Agudelo, B. 2019).

(Arévalo, J.; Bayona, R.; Rico, D. 2017) En definitiva, según Peláez J.; García y Azurero (2014), el talento humano necesita una transformación cultural que dimensione estrategias orientadas hacia un proyecto de vida del ser humano y el desarrollo de sus habilidades y talentos; lo que implicaría evolucionar hacia un modelo actual con un recurso calificado, $y$ fundamentalmente motivado y comprometido con la gestión de la organización.

\section{Conclusiones}

Definitivamente, el elemento más importante para el éxito de una organización es el talento con que cuente y la gestión adecuada, que permitirá que esté preparado, empoderado y propietario de su trabajo como proyecto de vida.

Desde el punto de vista metodológico, el enfoque bibliográfico utilizado, permite justificar el planteamiento base del artículo, en cuanto a que se debe determinar el uso adecuado de la gestión del talento; de ahí que una mala gestión de este primordial elemento se verá evidenciada en un potencial fracaso de la organización. Esta exploración básicamente bibliográfica busca aportar nuevos conocimientos que coadyuven a la solución de un problema latente, patentizado en una mala gestión de los colaboradores de una organización.

Debemos ser enfáticos en el conocimiento de la gestión humana y su impacto 
social en cualquier entorno. Inclusive la escasez de talento puede estar ligada a que los colaboradores no sientan como un reto el objetivo-fin de la organización.

La inversión en desarrollo, formación y sociabilidad del talento, donde este pueda retroalimentarse con ideas, opiniones, sueños y metas, es la inversión más sabia que cualquier organización pueda realizar. Estas actividades contribuirán a que el talento enfrente los cambios imprescindibles para continuar avanzando en la consecución de metas cada vez más anhelosas y retadoras.

Si los talentos de una organización se sienten satisfechos y empoderados con sus tareas y responsabilidades, el ritmo de producción estará garantizado y la evaluación integral será esperada como un instrumento para remediar fallas, permitiendo la flexibilidad necesaria en espacios donde se requiere, y así facilitar el desarrollo exitoso de las actividades asignadas.

En toda empresa siempre existirán colaboradores acaparadores de "poder", que no se podrá prescindir de su talento, pues mas bien se deberá permitirles colaborar en todos los niveles de la organización. El talento referido evidencia varios elementos coadyuvantes, tales como el conocimiento, la experiencia, la incentivación reflejada en motivación, intereses vocacionales, aptitudes, actitudes, habilidades, potencialidades, salud, entre otros.

\section{Referencias bibliográficas}

Ávila, B. (s.f.). Comunicación no verbal: la regla Mehrabian. Disponible en: https://www. gestion-comercial.com/comunicacionno-verbal-la-regla-mehrabian/

Chiaveanato, I. (2013). Gestión del talento humano (3. ${ }^{\circ}$ ed.) McGraw-Hill Interamericana S.A. ISBN 978-970-10-7340-7

Llácer, P. (27 de febrero de 2019). La gestión del talento, la clave de nuestro futuro. México. Disponible en: https://www.forbes.com. $\mathrm{mx} /$ la-gestion-del-talento-la-clave-denuestro-futuro/

Mehrabian, A. (2008). Noverbal Communication, Editorial Aldine-Atherton. Disponible en: https://www.gestion-comercial.com/comunicacion-no-verbal-la-regla-mehrabian/

Rodríguez Cruz, Y. (2021). Gestión del capital humano y del talento humano ¿son iguales? Bogotá, Colombia: Gestiopolis. Disponible en https://www.gestiopolis.com/gestiondel-capital-humano-y-del-talento-humano-son-iguales/

Sukier, H., Molina R., Parra M, Cueto, K.(2020). Gestión estratégica de talento humano desde un enfoque sustentable, 9(36). Disponible en: http://portalvirtualempleo. us.es/talento/

Claussen, J; Grohsjean, F.; Luger J.; Probst, G. (2014). Talent management and careerdevelopment: What it takes to get promoted. Journal of World

González, T.; Martínez, C.; Pardo Del Val, M. (2009). La gestión del talento en la empresa industrial española. Economía industrial, ISSN 0422-2784 No.374 págs. 21-35

Universidad de Sevilla, Profesionales (2020). El Talento, más allá del conocimiento y la experiencia. Noviembre 10: http://portalvirtualempleo.us.es/talento/

Bell, R., Espín, M., Espín, E (2015). Revista Científica ECOCIENCIA, Tendencias de la Gestión de Talento Humano en el sector público.

Benítez, K. (2005). Consideraciones sobre la gestión del talento Humano. El enfoque del mercado interno. Revista Visión Gerencial. (Universidad de Los Andes). Mérida. ISSN:1317-8822 VOL. 4

Piñango, R. (2004). Después de todo se trata de gente. Editorial a la Edición Debates IESA. Volumen X. Número 1. Octubre-Diciembre 2004,4

Goleman, D. (2010). La inteligencia emocional en la empresa, España. Zeta 464ISBN 10: 987.

Berta E. Madrigal Torres (2009). Habilidades Directivas, Segunda Edición. McGraw-Hill. ISBN 13: 978- 970-10-6875-5 / ISBN 10: 970-10-6875-0

Vivas, E (2010). El ser humano y la organización. Técnica de procesos. Buenos Aires: 
https://comunidad.iebschool.com/giulianapsicologia/36-2/

Guillen, C., \& Guil, R. (2007). Psicologia del trabajo para relaciones laborales. Madrid: MC Graw Hill.

Ferrer, A. (2008). Recursos Humanos: Una mirada al centro organizacional: http:// ve.scielo.org/scielo.php?script=sci_arttex t\&pid=\$1010-29142016000200008

Agudelo, B. (2019). Formación del talento humano y la estrategia organizacional en empresa de Colombia. ISSN 1900-3803/eISSN 2539-0279. Vol.15

Arévalo, J., Bayona. R., Rico., D.(2017), Las prácticas innovadoras de la gestión humana: Una necesidad estratégica e las microempresas. Revista Ciencias Estratégicas.
Enero-junio Vol.25, no.37. p. 15-24. DOl: http://dx.doi.org/10.18566/rces.v25n3

Peláez, J., García, M., y Azuero, A. (2014). La relación estratégica entre la gestión humana y la responsabilidad social empresarial: avances de una explicación en un caso colombiano. Suma de Negocios, 5(11), 1528. Recuperado de http://www.elsevier. es/en-revista-suma-negocios-208-articulo-la-relacion-estrategica-entre-gestion-90375922

Bayraktar, C. Hancerioguillari, G., Cetinguc, B., Calisir, F., Competitive strategies, innovation, and firm performance: an empirical study in a developing economy environ-ment. In: Technology Anlysis \& Strategic Management 2017.vol.29, no.1, p.38-52.DOl: 10.1080/09537325.2016.119497 